ISSN 0103-5150

Fisioter. Mov., Curitiba, v. 25, n. 2, p. 273-280, abr./jun. 2012 Licenciado sob uma Licença Creative Commons

\title{
Prevalência e fatores associados à dor musculoesquelética em profissionais de atividades sedentárias ${ }^{1}$
}

\author{
Prevalence and factors associated with musculoskeletal \\ pain in professionals of sedentary activities
}

\begin{abstract}
Alberto de Vitta ${ }^{[a]}$, Alessandra Aparecida Canonici ${ }^{[b]}$, Marta Helena Souza de Conti ${ }^{[c]}$, Sandra Fiorelli de Almeida Penteado Simeão ${ }^{[\mathrm{d}]}$

[a] Professor Doutor do curso de Fisioterapia e do Mestrado em Saúde Coletiva da Universidade do Sagrado Coração (USC), Bauru, SP - Brasil, e-mail: albvitta@yahoo.com.br

[b] Graduada em Fisioterapia pela Universidade do Sagrado Coração (USC), Bauru, SP - Brasil, e-mail: canonici@yahoo.com.br

[c] Professora Doutora do curso de Fisioterapia e do Mestrado em Saúde Coletiva da Universidade do Sagrado Coração (USC), Bauru, SP - Brasil, e-mail: madeconti@yahoo.com.br

[d] Professora Doutora do Mestrado em Saúde Coletiva da Universidade do Sagrado Coração (USC), Bauru, SP - Brasil, e-mail: ssimeao@usc.br
\end{abstract}

\section{Resumo}

Objetivos: Verificar a prevalência dos sintomas musculoesqueléticos e investigar fatores associados, em funcionários de uma empresa de prestação de serviço de fornecimento de água e tratamento de esgoto da cidade de Bauru, São Paulo. Materiais e métodos: Trata-se de um estudo transversal, com 176 profissionais que exerciam atividades sedentárias e que responderam a questionários para coleta de dados das variáveis demográficas, ocupacionais e hábitos de vida, o Índice de Capacidade para o Trabalho e o Questionário Nórdico de Sintomas Osteomusculares. Foram realizadas análises descritiva, bivariada e multivariada por regressão logística binária. Resultados: Os trabalhadores relataram algum tipo de sintoma osteomuscular nos últimos 12 meses, principalmente na região lombar (40,3\%), na coluna cervical (27,2\%) e nos ombros $(23,8 \%)$. Observou-se associação entre os sintomas osteomusculares e as seguintes variáveis: tipo de movimento, postura no trabalho e problemas de saúde. Conclusão: Conclui-se que a prevalência de sintomas osteomusculares nos trabalhadores é alta e que houve uma associação significativa com os movimentos repetitivos, postura

\footnotetext{
1 Instituição de fomento: PIBIC/CNPq.
} 
sentada e problemas de saúde, sendo, portanto, necessária uma atuação interdisciplinar, multiprofissional e intersetorial, de forma a interferir positivamente no processo de trabalho e na saúde do trabalhador.

Palavras-chave: Saúde do trabalhador. Sintomas musculoesqueléticos. Condições de trabalho. Atividades sedentárias.

\begin{abstract}
Objectives: To determine the prevalence of musculoskeletal symptoms and to investigate factors associated with employees of a company that provides service of water supply and sewage treatment in the city of Bauru, São Paulo. Materials and methods: This is a cross-sectional study with 176 professionals who perform sedentary activities and answered questionnaires for data collection of demographic, occupational and lifestyle variables, the Index of the Work Ability and the Nordic Musculoskeletal Questionnaire. Descriptive analysis, bivariate and multivariate binary logistic regression were made. Results: The workers reported some type of musculoskeletal symptoms in the last 12 months, mainly in the lumbar region (40.3\%), cervical spine (27.2\%) and shoulders (23.8\%). There was an association between musculoskeletal symptoms and the variables type of movement, posture at work and health problems. Conclusion: The prevalence of musculoskeletal symptoms in workers is high and there was a significant association with repetitive movements, sitting posture and health problems, being therefore, needed an interdisciplinary, intersectoral and multidisciplinary presence, in order to positively affect the process of work and occupational health.
\end{abstract}

Keywords: Workers health. Musculoskeletal pain. Work condition. Sedentary activities.

\section{Introdução}

0 trabalho tem um importante papel na vida do homem, pois, além de ser fonte do seu sustento, nele pode sentir-se útil, produtivo e valorizado. Entretanto, quando realizado em condições inadequadas, pode ser nocivo, prejudicando a saúde, provocando doenças, levando à inatividade, encurtando a vida e até causando a morte $(1,2)$.

Diversas profissões são estudadas, evidenciando-se a necessária identificação de riscos e condicionantes ergonômicos presentes nos ambientes de trabalho. As atividades ocupacionais sedentárias são citadas em virtude de ocorrência de posturas inadequadas, tarefas repetitivas e monótonas, excesso de horas trabalhadas e ausência de pausas, mobiliário e equipamentos inadequados, dificuldade de relacionamentos interpessoais, pressão da chefia, dentre outros (3).

Os diversos fatores geram alterações nas estruturas musculoesqueléticas da coluna lombar (aumento da pressão interna no núcleo do disco intervertebral, estiramento dos ligamentos, pequenas articulações e nervos), reduz a circulação de retorno dos membros inferiores e promove desconfortos na região do pescoço e membros superiores (4).
O conjunto de alterações nas estruturas músculos-esqueléticas representa um sério problema para o indivíduo e, mesmo de saúde pública, visto ser uma das mais importantes causas de incapacidades e absenteísmo em trabalhadores (5).

Estudos transversais descrevem a prevalência de sintomatologia musculoesquelética em atividades sedentárias. Brandão et al. (6) avaliaram 502 bancários de Pelotas e região, e notaram que $60 \%$ destes mencionaram dor musculoesquelética no último ano. Prati et al. (7) verificaram que 78,7\% dos digitadores de um jornal de Porto Alegre apontaram sintomas musculoesqueléticos. No estudo de Kose (8) com taquígrafos da Câmara Municipal de São Paulo, a prevalência foi de $85 \%$.

A relevância de estudos que visam explorar a associação direta e indireta entre demanda física no trabalho e as variáveis pessoais, físicas, psicológicas, sociais e do meio ambiente em diferentes ocupações residem no fato de que essas patologias geram consequências sociais e econômicas, tanto para as empresas como para o Estado. Para a empresa, significa a redução do número de homens/horas trabalhado, em razão da ausência de atividade por períodos de tempo consideráveis, o que provoca uma perda na produtividade e na qualidade do serviço. Para o 
Estado, as despesas recaem sobre o INSS e decorrem do pagamento de benefícios previdenciários. Além do benefício pago ao acidentado ou dependente, as despesas de tratamento e reabilitação são de responsabilidade do INSS. No Brasil, o volume de recursos destinados ao pagamento de benefícios decorrentes de acidentes de trabalho ou doenças ocupacionais acarreta despesas de cerca de $\mathrm{R} \$ 2,1$ bilhões ao ano à Previdência Social. Pode-se notar que são gastos elevados e que poderiam ser utilizados de outra maneira (9).

Investigações nesses domínios podem ser utilizadas como apoio para os profissionais do Serviço de Saúde Ocupacional de empresas em suas avaliações e estudos sobre o trabalho e os fatores que os afetam, além de oferecer subsídios para medidas de reabilitação e proposição de programas preventivos, que contribuirão para a melhoria da qualidade de vida dos trabalhadores e o aumento de suas chances de uma velhice satisfatória (10).

Para contribuir na investigação da prevalência de sintomas musculoesqueléticos e dos efeitos das variáveis demográficas, relativas às condições de trabalho e saúde geral, delineou-se o presente estudo com o objetivo de verificar a prevalência dos sintomas musculoesqueléticos e investigar fatores associados em funcionários de uma empresa de prestação de serviço de fornecimento de água e tratamento de esgoto da cidade de Bauru.

\section{Materiais e métodos}

Realizou-se um estudo transversal com 210 funcionários de uma empresa de prestação de serviço de fornecimento de água e tratamento de esgoto da cidade de Bauru, que exerciam atividades sedentárias.

Desse total, foram sujeitos 176 profissionais, do sexo masculino, que utilizavam a postura sentada ou alternada (ora sentado, ora em pé), utilizavam microcomputador e nas suas atividades realizavam tarefas como digitação de documentos e dados, leitura e escrita de documentos e atendimento ao público com preenchimento de documentos. As perdas foram em virtude de férias e do desinteresse em responder os questionários durante o estudo.

O critério para classificar os sujeitos como sedentários no trabalho foi a estimativa de consumo calórico exigido no trabalho, proposto por Couto (11). Também, nesse estudo, foi controlada a variável envolvimento em atividade ocupacional sedentária, ou seja, os sujeitos estavam trabalhando nessas atividades há mais de um (1) ano e permaneciam executando essas atividades por, pelo menos, metade de sua jornada de trabalho.

A coleta de dados foi realizada nos diversos setores da empresa, onde se alocavam os funcionários, pelos pesquisadores do curso de graduação em Fisioterapia da Universidade do Sagrado Coração, em Bauru. Foram explicados, individualmente, os objetivos da pesquisa, a não obrigatoriedade em participar e o caráter de sigilo dos dados. Em seguida, foram apresentados os questionários e solicitado o autopreenchimento imediato dos mesmos. Todo esse processo ocorreu entre fevereiro e abril de 2007.

Foi utilizado um questionário multidimensional composto dos seguintes itens:

1) Aspectos sociodemográficos: idade, estado civil, grau de instrução;

2) Condições de trabalho: cargo na empresa, tempo que trabalha na empresa, carga horária de trabalho, realização de pausas além do almoço, principal postura no trabalho, número de horas na principal postura, tipo de movimento e falta ao trabalho;

3) Saúde geral: problemas de saúde, nível de atividade física e tabagismo.

Também foi avaliada a capacidade para o trabalho por meio do questionário autoaplicável denominado Índice de Capacidade para o Trabalho (ICT) (12), adaptado para a população brasileira. É composto por 60 questões sobre aspectos pessoais, demanda física e mental no trabalho e estado de saúde do trabalhador.

Esse conjunto de variáveis (aspectos sociodemográficos, condições de trabalho, saúde geral e capacidade para o trabalho) foi considerado como independente.

Os sintomas musculoesqueléticos, considerados neste estudo como desfecho, foram medidos por meio do questionário Nórdico de Sintomas Osteomusculares, composto pelas seguintes questões: nos últimos 12 meses, você teve problemas (dor, formigamento/ dormência) nas regiões específicas do corpo (pescoço, ombro, cotovelo, punho e mão, coluna dorsal, cervical, lombar, quadris, coxas e nádegas, joelhos, tornozelos e pés)?; nos últimos 12 meses, você foi impedido de realizar atividades normais (trabalho, atividades 
domésticas e de lazer) por causa desse problema nas regiões citadas acima?; nos últimos 12 meses, você consultou algum profissional da área da saúde (ex.: médico, fisioterapeuta) por causa dessa condição?; nos últimos sete dias, você teve algum problema (dor, formigamento/dormência) nas regiões corporais?. Esse instrumento foi adaptado culturalmente para a língua portuguesa por Barros e Alexandre (13), apresentando uma confiabilidade que varia de 0,88 a 1, segundo o coeficiente de Kappa, validado por Pinheiro, Tróccoli e Carvalho (14) com um índice adequado de validade para a versão brasileira.

Os dados obtidos foram introduzidos em um banco de dados do programa estatístico SPSS (versão 10.0). A análise foi realizada mediante uma abordagem descritiva e outra analítica. Na abordagem descritiva, foi feita a distribuição de frequência absoluta e relativa para variáveis categóricas. $\mathrm{Na}$ abordagem analítica, realizou-se uma análise bivariada utilizando o teste do qui-quadrado de Pearson, para que fosse possível observar as possíveis associações entre as variáveis independentes com a variável dependente "presença de sintomatologia dolorosa". Em seguida, foi realizada análise multivariada, por regressão logística binária, utilizando análise hierarquizada. Mediante a estratégia estabelecida de associações entre as dimensões estudadas (sociodemográficas, condições de trabalho e condições de saúde), foram elaborados três modelos explicativos de regressão logística binária, introduzindo as variáveis em forma de blocos, na ordem acima descrita, permanecendo no modelo subsequente apenas as variáveis que tiveram significância estatística $(\mathrm{p}<0,05)$ no modelo anterior. 0 critério de saída para todas as variáveis introduzidas em cada modelo foi $\mathrm{p}<0,20$. Ao fim, chegou-se a um modelo final de regressão com apenas aquelas variáveis de maior significância estatística. 0 método de introdução das variáveis nos modelos adotados foi o backward stepwise. Considerou-se um nível de significância p < 0,05 e intervalo de confiança (IC) de 95\%, com cálculo dos odds ratios ajustados $(15,16,17)$.

A pesquisa foi aprovada pelo Comitê de Ética em Pesquisa da Universidade do Sagrado Coração, sob o protocolo n. 065 .

\section{Resultados}

Um total de 176 sujeitos do sexo masculino participou desta pesquisa, sendo 59,1\% na faixa etária de 20 a 35 anos e $40,9 \%$ na de 36 a 55 anos. Em relação à escolaridade, $67 \%$ possuíam o Ensino Fundamental completo e, quanto ao estado civil, $81,2 \%$ eram casados.

Analisando-se o tempo de atividade nas funções, observou-se que $53,4 \%$ estavam na empresa há mais de 10 anos. Durante a jornada de trabalho, 55,7\% passavam mais tempo em pé, e quanto ao tipo de movimento, $62,5 \%$ executavam, alternadamente, posturas estáticas associadas a movimentos repetitivos. Quanto ao tempo de permanência na mesma postura durante o período de trabalho, 60,2\% relataram que permaneciam mais de seis horas, enquanto que, $97,7 \%$ realizavam pausas para descanso.

Observou-se que $16,5 \%$ dos sujeitos se ausentaram das atividades laborais nos últimos 12 meses por sintomatologia dolorosa relacionada ao trabalho. As queixas principais foram dores na coluna e pernas, respectivamente.

Sobre a saúde geral, 38,1\% eram praticantes regulares de atividades físicas, $75 \%$ eram não fumantes e 63,1 afirmaram possuir alguma patologia ou distúrbio. Quanto à capacidade para o trabalho, 65,3 apresentaram índice baixo.

A prevalência de sintomatologia musculoesquelética encontrada foi de $63,1 \%$ predominantemente na região lombar $(40,3 \%)$, coluna cervical $(27,2 \%)$ e ombros $(23,8 \%)$, como observado na Tabela 1.

0 estudo da associação entre os fatores sociodemográficos e a variável dependente mostrou que a idade $(p=0,16)$, a escolaridade $(p=0,25)$ e o estado

Tabela 1 - Número e proporção de sintomas musculoesqueléticos durante a atividade profissional

\begin{tabular}{lrr}
\hline Localização & n.* & \% * \\
\hline Cervical & 46 & 27,2 \\
Ombros & 42 & 23,8 \\
Costas superior & 9 & 5,1 \\
Cotovelo & 20 & 11,4 \\
Punho/mãos & 24 & 13,6 \\
Lombar & 71 & 40,3 \\
Quadris/coxas & 19 & 10,8 \\
Joelhos & 22 & 12,5 \\
Tornozelos/pés & 13 & 7,3 \\
\hline
\end{tabular}

Fonte: Dados da pesquisa.

Legenda: * $=$ Um mesmo indivíduo pode relatar dor em mais de uma região corporal. 
civil $(\mathrm{p}=0,25)$ não apresentaram associações significativas no grupo estudado.

Em relação à associação entre os fatores relacionados ao trabalho e o relato de sintomatologia musculoesquelética, as variáveis tempo na função ( $\mathrm{p}=0,30)$, número de horas no trabalho $(\mathrm{p}=0,96)$, pausas $(p=0,12)$ e número de faltas $(p=0,25)$ não se mostraram estatisticamente significativas, enquanto que, o tipo de movimento $(\mathrm{p}=0,007)$ e posturas no trabalho ( $p=0,0001)$ apresentaram significância estatística, conforme verificado na Tabela 2 .

A associação entre os aspectos de saúde geral e a sintomatologia musculoesquelética, o nível de atividade física $(\mathrm{p}=0,81)$ e tabagismo $(\mathrm{p}=0,78)$ não apresentaram associações significativas no grupo estudado, enquanto que os problemas de saúde ( $p=0,0001$ ) e o índice de capacidade para o trabalho $(p=0,01)$ mostraram tais associações, de acordo com o observado na Tabela 3.

Os resultados da análise de regressão logística (Tabela 4) evidenciaram que o tipo de movimento $(\mathrm{p}=0,01)$ e os problemas de saúde $(\mathrm{p}=0,0001)$ mostraram associação de forma independente ao relato de sintomatologia musculoesquelética.

\section{Discussão}

Segundo os resultados obtidos, foi possível conhecer os sintomas musculoesqueléticos apresentados pelos trabalhadores de uma empresa pública de uma cidade do estado de São Paulo, em 2007. Esses resultados demonstraram que a prevalência de sintomatologia musculoesquelética é alta, ou seja, 63,1\% dos trabalhadores referiram sintomas nos últimos 12 meses, semelhantes aos achados em outros estudos $(6,7,14,18)$. Essas evidências apoiam-se no fato da utilização de um instrumento validado e referido por diversos estudos, principalmente em saúde do trabalhador, com destaque para categorias de motoristas, enfermeiros, dentistas e industriários (14).

Com relação às regiões anatômicas onde predominam os sintomas, constatou-se que as principais foram: coluna lombar $(40,3 \%)$, coluna cervical $(27,3 \%)$ e ombros (23,8\%). Comparativamente, Prati et al. (7) notaram que os digitadores de um jornal de Porto Alegre mencionaram dores com maior frequência nas regiões dos ombros, punho e antebraço. Silva e Juvêncio (18), em 2005, relataram que os trabalhadores administrativos de uma Universidade apontaram
Tabela 2 - Análise bivariada entre variáveis de condições de trabalho e presença de dor

\begin{tabular}{lcccccccc}
\hline & $\begin{array}{c}\text { Nenhuma/ } \\
\text { uma região }\end{array}$ & & \multicolumn{2}{c}{$\begin{array}{c}\text { Mais de } \\
\text { uma região }\end{array}$} & \\
\cline { 2 - 3 } & n. & $\%$ & & n. & $\%$ & p \\
\hline Tipo de movimento & & & & & \\
\hline Repetitivo & 16 & 24,2 & & 50 & 75,8 & 0,005 \\
Postura fixa & 49 & 44,5 & & 61 & 55,5 & 0,005 \\
Posição no trabalho & & & & & \\
\hline Sentado & 17 & 21,8 & 61 & 78,2 & 0,001 \\
Alternado & 48 & 49,0 & 50 & 51,0 & \\
\hline
\end{tabular}

Fonte: Dados da pesquisa.

Tabela 3 - Análise bivariada entre variáveis de condições de saúde e presença de dor

\begin{tabular}{lccccccc}
\hline & $\begin{array}{c}\text { Nenhuma/ } \\
\text { uma região }\end{array}$ & & \multicolumn{2}{c}{$\begin{array}{c}\text { Mais de } \\
\text { uma região }\end{array}$} & \\
\cline { 2 - 3 } & n. & $\%$ & & n. & $\%$ & & p \\
\hline Problemas de saúde & & & & & \\
\hline Sim & 22 & 19,8 & & 89 & 80,2 & 0,001 \\
Não & 43 & 66,2 & 22 & 33,8 & \\
\hline Capacidade de trabalho & & & & & \\
\hline Baixa & 35 & 30,4 & 80 & 69,6 & 0,01 \\
Alta & 30 & 49,2 & 31 & 50,8 & 0 \\
\hline
\end{tabular}

Fonte: Dados da pesquisa.

Tabela 4 - Resultado da análise multivariada de regressão logística, modelo final para associações independentes com a presença de sintomatologia dolorosa

\begin{tabular}{|c|c|c|c|c|}
\hline Variáveis & Referência & $\mathrm{p}$ & $\mathrm{OR}_{\text {ajustado }}$ & IC $95 \%$ \\
\hline \multicolumn{5}{|c|}{ Problemas de saúde } \\
\hline Sim & Não & 0,0001 & 6,38 & $3,07-13,2$ \\
\hline \multicolumn{5}{|c|}{ Posição no trabalho } \\
\hline Sentado & Alternado & 0,004 & 3,40 & $1,59-7,26$ \\
\hline \multicolumn{5}{|c|}{ Tipo de movimento } \\
\hline Repetitivo & Postura fixa & 0,02 & 4,04 & $1,84-8,88$ \\
\hline
\end{tabular}

Fonte: Dados da pesquisa. 
as regiões da coluna lombar e ombros como os locais mais acometidos. Em um estudo com operadores de caixas de supermercado, Batiz, Santos e Licea (19) notaram que $81 \%$ apresentaram dores na região lombar, $54 \%$ relataram dores nos ombros, $50 \%$ apresentaram dores na coluna dorsal e $49 \%$ no pescoço.

No presente estudo, evidenciou-se que os sintomas mais frequentes em ordem de importância foram na região lombar, nos joelhos e na cervical. Estudos realizados por Armstong (20), Ekberg (21) e Vieira (22) revelam que mais de $70 \%$ das pessoas que trabalham em atividades sedentárias sofrem dores nas regiões lombar e cervical. Juul-Kristensen e Jensen (23) relataram que os sintomas ocorreram mais frequentemente na região lombar em trabalhadores que utilizavam o computador como instrumento de trabalho.

A associação entre os fatores relacionados ao trabalho e relato de sintomatologia musculoesquelética revelou que o tipo de movimento no trabalho apresentou significância estatística.

Em relação ao tipo de movimento, pôde-se notar que os sujeitos que realizam, preferencialmente, movimentos repetitivos tiveram cerca de quatro vezes mais chances de apresentar mais de uma região corporal com sintomatologia dolorosa.

Os estudos epidemiológicos confirmam a relação dos movimentos de repetição na origem de muitos problemas musculoesqueléticos (24-26). Entre os mecanismos fisiopatológicos referidos na gênese desses problemas, englobam-se contrações contínuas e aumento de pressão intramuscular, interrupção do aporte sanguíneo e compressões de feixes nervosos, levando ao sofrimento muscular crônico (20). Essas condições parecem explicar tanto a associação com a exposição no trabalho, como outras atividades que envolvam esforço semelhante. Outros estudos mostraram que o trabalho repetitivo é associado com as dores no pescoço e nas costas com significância estatística (24). Andersen et al. (24) verificaram que indivíduos com elevados níveis de trabalho repetitivo tiveram um risco aumentado de dor no braço (OR 1,7 [IC 95\% 1,0-2,9]).

Os indivíduos cujo trabalho era realizado, principalmente na postura sentada, apresentaram três vezes mais chances de possuir dor em mais de um local. A postura sentada gera várias alterações nas estruturas musculoesqueléticas dos diversos segmentos corporais: aumenta em, aproximadamente, $35 \%$ a pressão interna no núcleo do disco intervertebral, estira todas as estruturas (ligamentos, pequenas articulações e nervos) da coluna vertebral, reduz a circulação de retorno dos membros inferiores e promove o desenvolvimento de processos inflamatórios nas estruturas osteomusculares com sintomatologia dolorosa associada $(4,9)$.

Eltayeb et al. (27) avaliaram 264 trabalhadores administrativos e notaram associação estatisticamente significativa entre os que permanecem por longos períodos na postura sentada. Andersen et al. (24) notaram que, nos trabalhadores estudados, não houve associação ente tipo de postura e sintomas musculoesqueléticos (OR 0,7 [IC 95\% 0,5-1,1]).

Na associação entre os aspectos de saúde geral e a sintomatologia musculoesquelética, notou-se que os problemas de saúde mostraram associações estatisticamente significativas. Os indivíduos portadores de algum problema patológico apresentaram seis vezes mais chances de possuir dor em mais de um local. As experiências patológicas são fatores de risco importantes, tornando os indivíduos mais propensos a sentirem dor $(1,28,29)$.

Andersen et al. (24) notaram que doenças crônicas foram significativamente associadas com a piora da dor em todas as regiões, sendo mais pronunciada no braço (OR 1,6 [IC 95\% 1,0-2,4]) e na parte inferior da perna (OR 1,7 [IC 95\% 1,2-2,4]).

As demais variáveis não apresentaram resultados estatisticamente significativos; no entanto, a literatura enquadra as mesmas como fatores de risco para a presença de dor relacionada ao trabalho $(1,5,6)$.

Este estudo possibilitou constatar a situação de saúde atual, dentro das dimensões estudadas, dos funcionários de uma empresa pública de uma cidade do estado de São Paulo, e sua relação com a sintomatologia dolorosa. Isso foi possível pela eleição do tipo de pesquisa, pois os estudos transversais possibilitam uma reflexão sobre a situação encontrada no momento da avaliação, mostrando um retrato instantâneo da amostra estudada. No entanto, de acordo com Brandão, Horta e Tomasi (6), esse tipo de estudo apresenta uma limitação, na medida em que, quando se utilizam instrumentos de autopreenchimento, pode ocorrer o viés das medidas simultâneas e a possível interferência de fatores não controlados.

Nesse contexto, pode-se concluir que houve uma associação significativa com os movimentos repetitivos e os problemas de saúde, sendo, portanto, necessária uma atuação interdisciplinar, multiprofissional e intersetorial, de forma a interferir positivamente no processo de trabalho e na saúde do trabalhador. 


\section{Referências}

1. Maciel ACC, Fernandes MB, Medeiros LS. Prevalência e fatores associados à sintomatologia dolorosa entre profissionais da indústria têxtil. Rev Bras Epidemiol. 2006;9(1):94-102.

2. Mergener CR, Kehring RT, Traebert J. Sintomatologia músculo-esquelética relacionada ao trabalho e sua relação com qualidade de vida em bancários do Meio Oeste Catarinense. Saúde e Soc. 2008;17(4):171-81.

3. Guérin F, LaVille A, Daniellou, F. Compreender o trabalho para transformá-lo: a prática da ergonomia. São Paulo: Edgard Blücher; Fundação Vanzolini, 2001.

4. Zapater AR, Silveira DM, De Vitta A, Padovani CR, Silva, JPC. Postura sentada: a eficácia de um programa de educação para escolares. Cien Saúde Colet. 2004;9(1):191-9.

5. Santos BS Filho, Barreto SM. Atividade ocupacional e prevalência de dor osteomuscular em cirurgiões-dentistas de Belo Horizonte, Minas Gerais, Brasil: contribuição ao debate sobre distúrbios osteomusculares relacionados ao trabalho. Cad Saúde Pública. 2001;17(1):181-93.

6. Brandão AG, Horta BL, Tomasi E. Sintomas de distúrbios osteomusculares em bancários de Pelotas e região: prevalência e fatores associados. Rev Bras Epidemiol. 2005;8(3):295-305.

7. Prati C, Krumenauer R, Blaya Carolina, Bonamigo DR, Boneti C, Lopes MLL. Frequência de L.E.R./D.O.R.T: lesões por esforços repetitivos/distúrbios osteomusculares relacionados ao trabalho em digitadores de um jornal em Porto Alegre. Rev Pesq Méd. 1999; 33(1-2):34-8.

8. Kose JI. A organização do trabalho de taquígrafos parlamentares: um estudo sobre o desenvolvimento de LER/DORT (Lesões por esforços repetitivos/Distúrbios Osteomoleculares Relacionados ao Trabalho) - o serviço de taquigrafia como uma linha de montagem. São Paulo: [s.n.]; 2005.

9. Brasil. Ministério da Saúde. Aumento de casos de doenças no INSS. Brasília: Ministério da Saúde; 2008.

10. De Vitta A, Zapater AR, Campos RS, Padovani CR. Desconfortos musculoesqueléticos percebidos em trabalhadores de diferentes faixas etárias, gêneros e ocupações. Fisioter Mov. 2007;20(1):29-36.
11. Couto HA. Ergonomia aplicada ao trabalho: manual técnico da máquina humana. Belo Horizonte: ERGO; 1995.

12. Tuomi K, Ilmarinen J, Jahkola A, Katajarinne L, Tulkki A. Índice de capacidade para o trabalho. Helsinki: Instituto Finlandês de Saúde Ocupacional; 1997.

13. Barros ENC, Alexandre NMC. Cross-cultural adaptation of the Nordic musculoskeletal questionnaire. Int Nurs Review. 2003;50(2):101-8.

14. Pinheiro FA, Tróccoli BT, Carvalho CV. Questionário nórdico de sintomas osteomusculares: validade do instrumento como medida de morbidade. Rev Saúde Pública. 2002;36:307-12.

15. Zar JH. Biostatistical analysis. 4th ed. New Jersey: Prentice-Hall; 1999.

16. Barros AJD, Hirakata V. Alternatives for logistic regression in cross sectional studies: an empirical comparison of models that directly estimate the prevalence ratio. BMC Med Res Methodol. 2003;3:21.

17. Ekman AG, Andersson EM, Hagberg M. Analyzing musculoskeletal neck pain, measured as present pain and periods of pain, with three different regression models: a cohort study BMC Musculoskelet Disord. 2009;10:73.

18. Silva CD, Juvêncio JF. Fatores humanos gerais e a aptidão física relacionada à saúde como agentes potencializadores de LER/DORT em trabalhadores de escritório. Rev Min Educ Fís. 2005;13(1):111-29.

19. Batiz EC, Santos AF, Licea OEA. A postura no trabalho dos operadores de checkout de supermercados: uma necessidade constante de análises. Prod. 2009; 19(1):190-201.

20. Armstong TJ, Bucke P, Fine LJ, Hagberg M, Jonsson B, Kilbom A, et al. A conceptual model for work-related neck and upper-limb musculoskeletal disorders. Scand J Work Environ Health. 1993;19(2):73-84.

21. Ekberg K, Bjorkqvist B, Malm P, Bjerre-Kiely B, Karlsson M, Axlson O. Casecontrol study of risk factors for disease in the neck and shoulder area. Occup Environ Med. 1994;51(4):262-6.

22. Vieira ER, Kumar S. Working postures: a literature review. J Occup Rehabil. 2004;14(2):143-59. 
23. Juul-Kristensen B, Jensen C. Self-reported workplace related ergonomic conditions as prognostic factors for musculoskeletal symptoms: The "BIT" follow up study on office workers. Occup Environ Med. 2005; 62(3):188-94.

24. Andersen JH, Haahr JP, Frost P. Risk factors for more severe regional musculoskeletal symptoms: a twoyear prospective study of a general working population. Arthritis Rheum. 2007;56(4):1355-64.

25. Vernaza-Pinzón P, Sierra-Torres CH. Dolor músculo-esquelético y su asociación con factores de riesgo ergonómicos en trabajadores administrativos. Rev Salud Publica. 2005;7(3):317-26.

26. Hannan LM, Monteilh CP, Gerr F, Kleinbaum DG, Marcus M. Job strain and risk of musculoskeletal symptoms among a prospective cohort of occupational computer users. Scand J Work Environ Health. 2005;31(5):375-86.
27. Eltayeb SM, Staal JB, Hassan AA, Awad SS, de Bie RA. Complaints of the arm, neck and shoulder among computer office workers in Sudan: a prevalence study with validation of an Arabic risk factors questionnaire. Environ Health. 2008;7:33. doi:10.1186/1476-069X-7-33

28. Westgaard RH, Jansen T. Individual and work related factors associated with symptoms of musculoskeletal complaints, II. Different risk factors among sewing machine operators. Br J Ind Med. 1992;49(3):154-62.

29. Rocha LE, Debert-Ribeiro M. Work, health and gender: a comparative study on systems analysts. Rev Saúde Pública. 2001;35(6):539-47.

Recebido: 11/04/2011

Received: 04/11/2011

Aprovado: 02/10/2011

Approved: 10/02/2011 